\title{
Metabolic Requirement for Inorganic Phosphate by the Rabbit Proximal Tubule
}

\author{
EVIDENCE FOR A CRABTREE EFFECT
}

\begin{abstract}
Peter C. Brazy, Steven R. Gullans, Lazaro J. Mandel, and Vincent W. Dennis, Division of Nephrology and Department of Physiology, Duke University and Durham Veterans Administration Medical Centers, Durham, North Carolina 27710
\end{abstract}

A BSTRACT These studies examine the effects of acute changes in the availability of inorganic phosphate on the function of isolated proximal renal tubules from rabbit kidney. We removed phosphate from the extracellular fluids and measured fluid absorption rates in isolated perfused tubules and oxygen consumption rates in suspensions of cortical tubules. In proximal convoluted tubules, the selective removal of phosphate from the luminal fluid reduced fluid absorption rates from $1.11 \pm 0.12$ to $-0.01 \pm 0.08 \mathrm{nl} / \mathrm{mm}$. $\mathrm{min}$. This effect on fluid absorption was dependent on the presence of glucose transport and metabolism. The addition of phlorizin to the phosphate-free luminal fluid preserved fluid absorption rates $(1.12 \pm 0.12 \mathrm{nl} /$ $\mathrm{mm} \cdot \mathrm{min})$ as did the substitution of nonmetabolized $\alpha$-methyl D-glucopyranoside for glucose $(1.05 \pm 0.21$ $\mathrm{nl} / \mathrm{mm} \cdot \mathrm{min}$ ) or the addition of 2-deoxyglucose, an inhibitor of glycolysis, to the bathing medium $(1.01 \pm 0.15 \mathrm{nl} / \mathrm{mm} \cdot \mathrm{min})$. There was no effect on fluid absorption if phosphate was removed from the bath only. Additionally, removal of phosphate from the luminal fluid of proximal straight rather than convoluted tubules had no effect on fluid absorption rates. Oxygen consumption rates in suspensions of cortical tubules were reduced from $18.9 \pm 0.6$ to $10.6 \pm 0.6 \mathrm{nmol} \mathrm{O}_{2} / \mathrm{mg}$ tubular protein.min by the removal of phosphate from the medium. This inhibition was prevented by the substitution of $\alpha$-methyl D-glucopyranoside for glucose in the phosphate-free medium. The data indicate that under certain conditions, proximal convoluted tubules require the presence of phosphate in the luminal fluid to preserve tubular function. In the absence of intraluminal phosphate, glucose metabolism causes a reduction in both oxidative metab-

Received for publication 21 October 1981 and in revised form 12 March 1982. olism and fluid absorption. This response is analogous to the Crabtree effect and suggests limitations on the intracellular availability of inorganic phosphate.

\section{INTRODUCTION}

Intracellular inorganic phosphate $(\mathrm{Pi})^{1}$ is important for both glycolysis and oxidative phosphorylation. In glycolysis, $\mathrm{Pi}$ is a substrate for glyceraldehyde-3-phosphate dehydrogenase and stimulates the activities of hexokinase (1) and phosphofructokinase (2). In oxidative phosphorylation, cytosolic $\mathrm{Pi}$ may regulate the rate of mitochondrial respiration via its contribution to the phosphorylation potential, ATP/ADP $\times \mathrm{Pi}(3$, 4). Although the relationship between $\mathrm{Pi}$ and the regulation of energy metabolism under physiologic conditions is still being investigated, certain experiments have defined conditions in which glycolysis and oxidative phosphorylation may be limited by the availability of Pi, i.e., phosphate control. For example, the addition of glucose to a suspension of Ehrlich ascites tumor cells decreases the cellular content of $\mathrm{Pi}$ and ATP and increases ADP $(5,6)$. If cytosolic $\mathrm{Pi}$ is reduced sufficiently, the rates of glycolysis and oxygen consumption are inhibited. Similarly, intracellular $\mathrm{Pi}$ and ATP are reduced in rat liver and kidney cells exposed to fructose (7-9). These observations suggest that the incorporation of $\mathrm{Pi}$ into glycolytic substrates may limit the availability of Pi for oxidative phosphorylation (6). This metabolic phenomenon is called the Crabtree effect, which is a hexose-induced inhibition of glycolysis and mitochondrial respiration $(6,10)$. Although the exact mechanism of the Crabtree effect remains

\footnotetext{
${ }^{1}$ Abbreviations used in this paper: Jv, fluid absorption rate; $\mathrm{Pi}$, inorganic phosphate; $\mathrm{QO}_{2}$, oxygen consumption rate.
} 
uncertain, its occurrence is indicative of coupling between glycolysis and oxidative metabolism, possibly via some function of intracellular $\mathrm{Pi}$ (4).

This study examines transport and metabolism of proximal renal tubules under conditions designed to alter the availability of inorganic phosphate. We reasoned that the removal of $\mathrm{Pi}$ from the ambient fluids might reduce intracellular $\mathrm{Pi}$ and thereby inhibit energy metabolism and ion transport by the tubules. The data indicate that under specific conditions, the selective removal of $\mathrm{Pi}$ from the intraluminal fluid markedly reduces fluid absorption rates by proximal convoluted tubules. This inhibition is related to some aspect of glucose metabolism because tubular function was preserved by the inhibition of glucose entry with phlorizin, by substitution of a nonmetabolized sugar for glucose, or by the inhibition of glycolysis with 2-deoxyglucose. Similarly, in suspensions of renal cortical tubules, removal of $\mathrm{Pi}$ from the medium reduced oxygen consumption rates when glucose was present but not if alpha methyl D-glucopyranoside replaced glucose. We conclude that the luminal entry of phosphate in proximal convoluted tubules provides phosphate for metabolism and that under some conditions, limited entry of phosphate inhibits oxidative phosphorylation and ATP production. Moreover, glucose metabolism appears to reduce the availability of intracellular $\mathbf{P i}$.

\section{METHODS}

Isolated perfused tubules. Segments of proximal tubules from rabbit kidney were isolated and perfused in vitro according to described techniques (11). Young female New Zealand White rabbits (1-3 kg) were maintained on rabbit chow (Ralston Purina Co., St. Louis, MO) and water. Proximal convoluted segments were dissected from cortical slices in chilled serum $\left(4^{\circ} \mathrm{C}\right)$. No attempt was made to select either superficial or juxtamedullary nephrons, or early or late proximal convoluted segments. Proximal straight segments were selected from superficial nephrons only. We perfused the tubules in vitro with a continuously exchanging bathing medium consisting of commercial rabbit serum (M. A. Bioproducts, Walkersville, MD) or a similar fluid maintained at $37^{\circ} \mathrm{C}, \mathrm{pH} 7.35-7.45$, and isosmotic with the perfusion fluid. The luminal perfusate was a physiological fluid containing: sodium chloride, $105 \mathrm{mM}$; sodium bicarbonate, $25 \mathrm{mM}$; potassium chloride, $5 \mathrm{mM}$; magnesium sulfate, $1 \mathrm{mM}$; monosodium phosphate, $2 \mathrm{mM}$; calcium chloride $1.5 \mathrm{mM}$; sodium lactate, $10 \mathrm{mM}$; L-alanine, $5 \mathrm{mM}$; and Dglucose, $6-12 \mathrm{mM}$. If necessary, more sodium chloride was added to maintain osmolality at $\sim 300 \mathrm{mosmol} / \mathrm{kg} \mathrm{H}_{2} \mathrm{O}$. Bathing media other than rabbit serum, when used, were made directly from the corresponding perfusion fluids by adding $6 \mathrm{~g} / \mathrm{dl}$ defatted albumin (12) and enough calcium chloride for a final total calcium concentration of $3 \mathrm{mM}$. All perfusion fluids contained ${ }^{125}$ [I]-iothalamate (Abbott Diagnostics, Diagnostic Products, North Chicago, IL) as a marker of fluid absorption. The rate of fluid absorption (Jv) was measured using a constant volume pipet of $\sim 150 \mathrm{nl}$ and timed collection periods. Calculation of $\mathrm{Jv}(\mathrm{nl} / \mathrm{mm} \cdot \mathrm{min})$ was according to the following equation (13):

$$
\mathrm{Jv}=\operatorname{Vo}\left[\left(\mathrm{I}_{\mathrm{o}} / \mathrm{I}_{\mathrm{i}}\right)-1\right] / \mathrm{L}
$$

where $\mathrm{Vo}(\mathrm{nl} / \mathrm{min})$ is the collection rate; $\mathrm{L}(\mathrm{mm})$ is the length of tubule perfused; and $I_{i}$ and $I_{o}(\mathrm{cpm} / \mathrm{nl})$ represent the concentration of ${ }^{125}$ I-iothalamate in the perfusate and collected fluid, respectively.

Transport rates for D-glucose and alpha methyl D-glucopyranoside were determined using radioisotopes $\left({ }^{14} \mathrm{C}\right.$, New England Nuclear, Boston, MA). We measured the lumen-tobath fluxes $\left(J_{s} \mathrm{~b}\right)$ as the difference between the amount of solute delivered and the amount collected according to the balance equation:

$$
\mathrm{J}_{s}^{\mathrm{lb}}=\left(\mathrm{ViC}_{\mathrm{i}}-\mathrm{VoC}_{\mathrm{o}}\right) \times[\mathrm{S}] / \mathrm{CiL}
$$

where $\mathrm{J}_{\mathrm{s}}^{\mathrm{lb}}(\mathrm{pmol} / \mathrm{mm} \cdot \mathrm{min})$ is the unidirectional solute flux; $\mathrm{Vi}(\mathrm{nl} / \mathrm{min})$ is the perfusion rate; $\mathrm{C}_{\mathrm{i}}$ and $\mathrm{C}_{\mathrm{o}}(\mathrm{cpm} / \mathrm{nl}) \mathrm{rep}$ resent the concentration of ${ }^{14} \mathrm{C}$-hexose in the perfusate and collected fluid, respectively; and [S] (pmol/nl) is the chemical concentration of the hexose in the perfusion fluid.

Two basic experiments were used in the perfusion studies. In one protocol, tubules were perfused and bathed in our standard Pi-containing fluids for a 30-40-min equilibration period followed by three or four timed collections over the next 30-45 min to measure transport rates. After these control measurements, we replaced either the luminal perfusate or the bath with a Pi-free solution in which chloride replaced $\mathrm{Pi}$. We allowed $15 \mathrm{~min}$ for reequilibration and then collected timed samples over the next 45-60 min. Because ${ }^{32} \mathrm{PO}_{4}$ was present in the initial perfusate, the thoroughness of removing Pi from the perf usion fluid could be assessed by measurement of any residual radioisotopic $\mathrm{Pi}$ in the collected fluid. After replacing the initial $\mathrm{Pi}$-containing perfusate with one that was free of both cold and radiolabeled $\mathrm{Pi}$, the radioisotopic activity of $\mathrm{Pi}$ in the collected fluid indicated a $\mathrm{Pi}$ concentration of $<0.02 \pm 0.01 \mathrm{mM}$. As we reported (14), the entry of $\mathrm{Pi}$ from Pi-containing bathing media into Pi-free perfusates results in $\mathrm{Pi}$ accumulation of $0.04 \pm 0.01 \mathrm{mM}$ in the collected fluid. Thus, the luminal $\mathrm{Pi}$ concentration was reduced to a very low value, which is less than the apparent $K_{\mathrm{m}}(0.13$ $\mathrm{mM}$ ) for $\mathrm{Pi}$ uptake by brush-border membrane from rabbit kidney (15).

In the other protocol, tubules were perfused from the time of dissection with Pi-free perfusates. Thus, in contrast to the protocol described above, these tubules were exposed to $\mathrm{Pi}$ free luminal fluid during the rewarming and equilibration period that followed dissection. Again, we allowed 30-40 min for the initial equilibration and then collected three or four samples during the subsequent 30-45 $\mathrm{min}$.

Radioisotopic activity was measured by liquid scintillation spectrophotometry in Aquasol (New England Nuclear) plus $0.5 \%$ water by volume. Inorganic phosphate was measured by the phosphomolybdate method (Auto/Stat Kit, Pierce Chemical Co., Rockford, IL); glucose by the glucose oxidase method (Pierce Chemical Co.); $\mathrm{pH}$ and $\mathrm{pCO}_{2}$ by electrodes (Instrumentation Laboratory, Inc., Lexington, MA), and osmolality by freezing-point depression (Precision Systems Inc., Sudbury, MA). Alpha methyl D-glucopyranoside and 2-deoxy D-glucose were obtained from Pfanstiehl Laboratories, Inc., Waukegan, IL, and phlorizin was obtained from Pfaltz \& Bauer Inc., Stamford, CT.

Data from each tubule are reported as the mean of at least three collections for each set of experimental conditions and are expressed as the mean $\pm S E$ for the number of tubules studied. Statistical comparisons were made using the paired or unpaired Student's $t$ test, as indicated. 
Suspensions of cortical tubules. We prepared suspensions of separated renal tubules with open lumens from rabbit cortex according to the methods of Balaban et al. (16). Briefly, kidneys from female New Zealand White rabbits were flushed in situ with $80 \mathrm{mg} / \mathrm{dl}$ collagenase (Sigma type IV, Sigma Chemical Co., St. Louis, MO), excised, and the cortex was dissected. Cortical tubules were then dispersed, washed several times at $4^{\circ} \mathrm{C}$, and centrifuged on a cushion of Ficoll $(400,000 \mathrm{~mol} \mathrm{wt})$ to remove nonvital cells and cellular debris. The final pellet was resuspended to give a concentration of 3-5 mg of tubule protein per milliliter in a solution that was identical to our synthetic perfusate except that it contained $0.6 \mathrm{~g} / \mathrm{dl}$ dialyzed dextran $(40,000 \mathrm{~mol} w \mathrm{t}$, Pharmacia Fine Chemicals, Uppsala, Sweden). If the hexose or $\mathrm{Pi}$ content of the media was altered, the tubules were exposed to the modified medium from the time of dissection at $4^{\circ} \mathrm{C}$ and were maintained in this medium during all subsequent procedures.

Suspensions of cortical tubules were preincubated for $30-$ $35 \mathrm{~min}$ at $37^{\circ} \mathrm{C}$ in a shaker bath and were bubbled with $95 \%$ $\mathrm{O}_{2} / 5 \% \mathrm{CO}_{2}$ to maintain the $\mathrm{pH}$ between 7.35 and 7.45. Oxygen consumption rates $\left(\mathrm{QO}_{2}\right)$ were measured polarographically in a closed $1.7-\mathrm{ml}$ chamber (16) using a Clark oxygen electrode (Yellow Springs Instrument Co., Yellow Springs, $\mathrm{OH})$. The oxygen tension in the suspension was recorded as a function of time and the slope reflected the rate of oxygen consumption. These rates were normalized per milligram of tubule protein (biuret method) in each suspension. Data are reported as the mean $\pm \mathrm{SE}$ for the number of suspensions examined. Control and experimental studies were performed on tubules obtained from the same kidney on the same day. Nystatin was obtained from Calbiochem-Behring Corp., (La Jolla, CA), and the compound 1799 was the gift of Dr. P. G. Heytler of DuPont Instruments-Sorval Biomedical Div., Newtown, CT.

\section{RESULTS}

Transport studies. The effect of Pi-free perfusate on $\mathrm{Jv}$ in proximal convoluted tubules depended on when the Pi was removed. For five proximal convoluted tubules studied with a Pi-free perfusate after preequilibration with $\mathrm{Pi}$-containing fluids, Jv was nor$\mathrm{mal}$ at $1.01 \pm 0.12 \mathrm{nl} / \mathrm{mm} \cdot \mathrm{min}$ during luminal perfusion with Pi-free fluids (Fig. 1A). In five other tubules, $\mathrm{Pi}$-free perfusate was introduced at the beginning of perfusion and was used throughout the initial equilibration period. For these tubules, Jv averaged $-0.01 \pm 0.08 \mathrm{nl} / \mathrm{mm} \cdot \mathrm{min}$ and was only partially restored by perfusion with a $\mathrm{Pi}$-containing perfusate for $1 \mathrm{~h}$ (Fig. 1B). Throughout all these studies, the bathing medium was normal rabbit serum, which contained $2.29 \pm 0.04 \mathrm{mM} \mathrm{Pi}$. Under these conditions, therefore, intraluminal $\mathrm{Pi}$ is required to maintain fluid absorption rates in proximal convoluted tubules, and the presence of $\mathrm{Pi}$ in only the bathing medium could not fulfill this requirement.

This profound reduction in $\mathrm{Jv}$ cannot be attributed solely to the elimination of phosphate-dependent sodium cotransport, which accounts for only a small portion of net sodium transport (14). We hypothesized instead that the reduction in $\mathrm{Jv}$ might be caused by

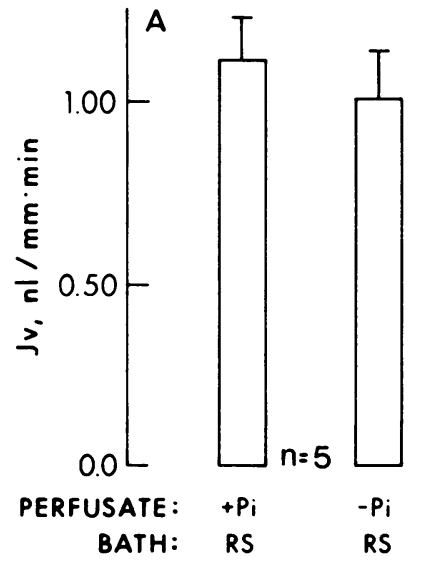

B

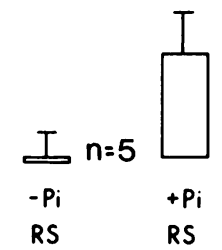

Figure 1 Effect on Jv of removing Pi from the perfusate. (A) Pi-free perfusate introduced after preequilibration with $\mathrm{Pi}$-containing fluids. Each bar represents the mean $\pm \mathrm{SE}$ for the $\mathrm{Jv}$ in five proximal convoluted tubules. The bathing medium was normal rabbit serum (RS). (B) Pi-free perfusate introduced at the outset of perfusion. Five proximal convoluted tubules were preequilibrated with a Pi-free perfusate, Jv was measured, and then a Pi-containing perfusate was begun. Jv was remeasured after $30 \mathrm{~min}$ of equilibration with the Pi-containing fluids.

an inhibition of energy metabolism related to limited availability of intracellular $\mathrm{Pi}$. Because glycolysis may reduce the content of intracellular $P i$ and $A T P(5,6)$, we examined the relationship between the reduction in $\mathrm{Jv}$ associated with a $\mathrm{Pi}$-free perfusate and glucose transport and metabolism. For these studies, proximal convoluted tubules were perfused from the beginning with a Pi-free perfusate and were bathed in normal rabbit serum, which contained $2.29 \mathrm{mM} \mathrm{Pi}$ and $6.09 \pm 0.42 \mathrm{mM} \mathrm{D}$-glucose. In the base-line condition with $6.5 \mathrm{mM}$ D-glucose in the Pi-free perfusate, Jv was zero and the glucose absorptive flux ${ }^{2}$ averaged $30.2 \pm 5.4$ $\mathrm{pmol} / \mathrm{mm} \cdot \mathrm{min}$ (Table I). The addition of phlorizin $(10 \mu \mathrm{M})$ to Pi-free perfusates that contained $6.8 \mathrm{mM}$ D-glucose resulted in complete inhibition of the glucose absorptive flux $(3.4 \pm 4.0 \mathrm{pmol} / \mathrm{mm} \cdot \mathrm{min})$ and preservation of the fluid absorption rate $(1.12 \pm 0.12$ $\mathrm{nl} / \mathrm{mm} \cdot \mathrm{min}$; Table I). These data indicate that the reduction in fluid absorption is not simply a consequence of removing intraluminal $\mathrm{Pi}$, but is related to some aspect of glucose entry into the cells. The data,

\footnotetext{
${ }^{2}$ Under these conditions, the glucose absorptive flux is phlorizin sensitive (see below) and presumably occurs via the sodium-dependent cotransport mechanism. This glucose transport rate is lower than the normal maximum rate but we are not certain whether these low rates reflect unsaturated transport $(17,18)$, axial heterogeneity for glucose transport (19), or inhibition by Pi-free conditions. Because of the degree of reduction in Jv, it seems likely that these are inhibited rates of glucose transport.
} 
TABLE I

Effects of Modifications in Glucose Transport on Jv during Perfusion with a Pi-free Perfusate

\begin{tabular}{|c|c|c|c|c|c|c|}
\hline Perfusate & Bath & Length & $v_{i}$ & Jv & [Gluc]o & $\begin{array}{c}\text { Hexose } \\
\text { absorptive flux }\end{array}$ \\
\hline & & $m m$ & $n l / \min$ & $n l / m m \cdot \min$ & $\mathrm{pmol} / \mathrm{nl}$ & $\mathrm{pmol} / \mathrm{mm} \cdot \min$ \\
\hline I Gluc $6.5 \mathrm{mM}, n=8$ & RS & $0.97 \pm 0.06$ & $13.78 \pm 1.49$ & $-0.02 \pm 0.05$ & $4.54 \pm 0.41$ & $30.2 \pm 5.4$ \\
\hline $\begin{array}{l}\text { II Gluc } 6.8 \mathrm{mM} \\
\text { Phlorizin } 10 \mu \mathrm{M} \\
n=4\end{array}$ & RS & $1.11 \pm 0.12$ & $16.48 \pm 1.08$ & $1.12 \pm 0.12$ & $7.16 \pm 0.22$ & $3.4 \pm 4.0$ \\
\hline III $\alpha$ MG $6.7 \mathrm{mM}, n=5$ & RS & $1.37 \pm 0.13$ & $16.60 \pm 1.47$ & $1.05 \pm 0.21$ & $5.67 \pm 0.87^{\circ}$ & $18.9 \pm 2.8^{\circ}$ \\
\hline IV Gluc $6.1 \mathrm{mM}, n=4$ & $\begin{array}{l}\mathrm{RS}+10 \mathrm{mM} \\
\text { 2-DG }\end{array}$ & $1.47 \pm 0.61$ & $16.19 \pm 1.33$ & $1.01 \pm 0.15$ & $3.35 \pm 1.02$ & $34.0 \pm 3.5$ \\
\hline
\end{tabular}

Values are means $\pm \mathrm{SE}$ for $n$, number of proximal convoluted tubules in each group. All tubules were studied with a Pi-free perfusate containing the noted concentration of sugar. The bath was normal rabbit serum (RS). Abbreviations are as follows: D-glucose, Gluc; $\alpha$-methyl D-glucopyranoside, $\alpha \mathrm{MG}$; 2-deoxyglucose, 2-DG; perfusion rate, Vi; fluid absorption rate, Jv; and glucose concentration in collected perfusate, [Gluc]o.

- Indicates values determined for $\alpha \mathrm{MG}$ instead of Gluc.

however, do not indicate whether the reduction in Jv is related to the transport of glucose or its metabolism.

To distinguish between these two possibilities, we examined the effects of $\mathrm{Jv}$ of replacing D-glucose in the Pi-free perfusate with alpha methyl D-glucopyranoside, a transported but nonmetabolized sugar. In the presence of $\mathrm{Pi}$, the substitution of $\alpha$-methyl D-glucopyranoside $(7 \mathrm{mM})$ for D-glucose had no effect on $\mathrm{Jv}(1.03 \pm 0.17$ vs. $1.00 \pm 0.14 \mathrm{nl} / \mathrm{mm} \cdot \min ; n=5)$. As shown in Table I, the substitution of $\alpha$-methyl D-glucopyranoside for $\mathrm{D}$-glucose in a $\mathrm{Pi}$-free perfusate prevented the inhibition of $\mathrm{Jv}(1.05 \pm 0.21 \mathrm{nl} / \mathrm{mm} \cdot \mathrm{min})$. Under these conditions, the absorptive flux of $\alpha$-methyl D-glucopyranoside averaged $18.9 \pm 2.8 \mathrm{pmol} / \mathrm{mm}$. min, which was not different from its absorptive flux in the presence of $\mathrm{Pi}(17.3 \pm 2.3 \mathrm{pmol} / \mathrm{mm} \cdot \mathrm{min} ; n$ $=8$ ). Thus, in contrast to glucose, transport of a nonmetabolized hexose does not reduce $\mathrm{Jv}$ in the presence of a $\mathrm{Pi}$-free perfusate.

Further experiments examined the influence of glucose metabolism by adding 2-Deoxyglucose (10 $\mathrm{mM}$ ) to the bathing medium of proximal convoluted tubules perfused with Pi-free intraluminal fluid. ${ }^{3}$ 2-Deoxyglu-

\footnotetext{
${ }^{3}$ In the presence of phosphate, the addition of 2-deoxyglucose (10 $\mathrm{mM}$ in bath and lumen) had no effect on Jv $(0.90 \pm 0.17$ vs. $1.01 \pm 0.12 \mathrm{nl} / \mathrm{mm} \cdot \mathrm{min})$. To the extent that 2-deoxyglucose inhibits glycolysis, these data indicate that glycolysis is not necessary to provide adequate metabolic energy to sustain net sodium transport when $10 \mathrm{mM}$ lactate and other substrates are available. Another inhibitor of glycolysis, however, iodoacetic acid $(0.1 \mathrm{mM}$ in bath), caused a marked inhibition of $\mathrm{Jv}(0.71 \pm 0.08$ to $0.15 \pm 0.04 \mathrm{nl} / \mathrm{mm}$. min), which we attribute to iodoacetic acid's lack of specificity.
}

cose readily enters renal tubular cells $(20)$, undergoes phosphorylation by hexokinase, and then competitively inhibits further glycolysis of glucose-6-phosphate at the phosphoglucose isomerase enzyme (21). In the presence of 2-deoxyglucose and the absence of intraluminal $\mathrm{Pi}, \mathrm{Jv}$ averaged $1.01 \pm 0.15 \mathrm{nl} / \mathrm{mm} \cdot \mathrm{min}$ and the glucose absorptive flux averaged $34.0 \pm 3.5$ $\mathrm{pmol} / \mathrm{mm} \cdot \mathrm{min}$, which was not different from the base-line glucose transport rate (Table I). Thus, 2deoxyglucose prevents the reduction in $\mathrm{Jv}$ by a $\mathrm{Pi}$-free perfusate presumably by its action on glucose metabolism rather than transport because 2-deoxyglucose had no demonstrable effect on the glucose absorptive flux.

For the data shown in Fig. 1A, the preequilibration of tubules with $\mathrm{Pi}$-containing fluids presumably restored or preserved the availability of intracellular $\mathrm{Pi}$. Under these conditions more glucose metabolism may be required to reduce significantly the tubular content of $\mathrm{Pi}$. To test this possibility, we attempted to increase glucose metabolism by increasing the rate of glucose transport. In this regard, it is useful to note that glucose transport in the proximal convoluted tubule increases as a function of glucose delivery over the range from 0 to $200 \mathrm{pmol} / \mathrm{min}(17,18,22)$. Accordingly, we examined the effect on Jv of increasing the glucose delivery rate in those tubules preequilibrated with $\mathrm{Pi}$ containing fluids before the removal of $\mathrm{Pi}$ from the perfusate. Fig. 2 shows fluid absorption rates as a function of time for five tubules perfused with $6.34 \mathrm{mM}$ D-glucose at a mean delivery rate of $84.0 \pm 6.7 \mathrm{pmol} /$ min (solid line) and for five tubules perfused with $12.66 \mathrm{mM} \mathrm{D}$-glucose at a mean delivery rate of $168.2 \pm 6.6 \mathrm{pmol} / \mathrm{min}$ (dashed line). After equilibration 


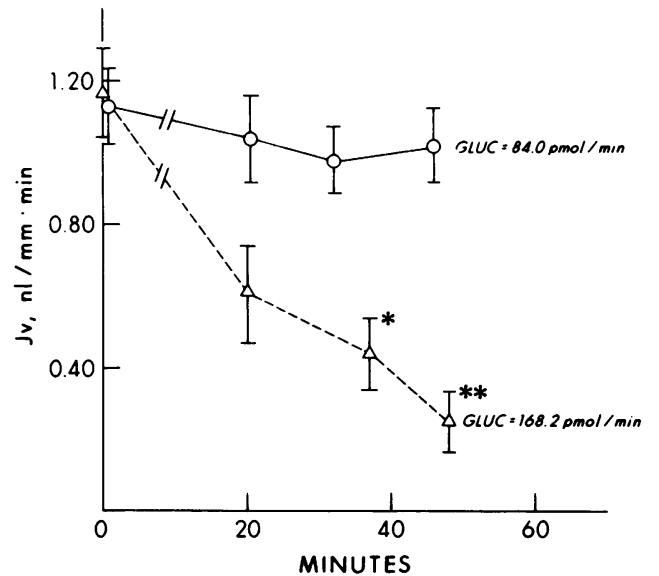

Figure 2 Effect on Jv of time and glucose delivery rate in absence of intraluminal $\mathrm{Pi}$. Two groups of proximal convoluted tubules were perfused by the protocol shown in Fig. 1A. At $\mathrm{t}=0$ the $\mathrm{Pi}$-containing perfusate was replaced with a $\mathrm{Pi}$-free fluid. In five tubules the perfusate concentration of glucose was $6.34 \mathrm{mM}$ and the mean $\pm S E$ value of $J v$ is represented by the open circles and solid line $(\mathrm{O}-\mathrm{O})$. In five other tubules, the glucose concentration was 12.66 $\mathrm{mM}$ and the mean value of $\mathrm{Jv}$ is indicated by the open triangles and broken line $(\Delta---\Delta)$. The break in the lines represents interruptions in the measurements necessitated by changing the intraluminal fluid. The bathing medium was normal rabbit serum. The average glucose delivery rate (Gluc) is indicated on the Figure. Statistics are the unpaired $t$ test with ${ }^{\bullet}$ denoting $P<0.05$ and $\cdots P<0.01$.

for $\sim 60$ min with Pi-containing perfusates, the mean values for $\mathrm{Jv}$ in the presence of $\mathrm{Pi}$ were similar $(1.13 \pm 0.19$ vs. $1.17 \pm 0.13 \mathrm{nl} / \mathrm{mm} \cdot \mathrm{min})$ at each rate of glucose delivery. At $t=0$ in Fig. 2, each group was perfused intraluminally with Pi-free fluid. With $\mathrm{Pi}$ free perfusate, $\mathrm{Jv}$ decreased steadily in those tubules perfused at the higher glucose delivery rates but was preserved in tubules perfused at lower rates of glucose delivery. These data indicate that the rate of glucose delivery and subsequent transport affect the tubule's requirement for intraluminal $\mathbf{P i}$.
We also examined the effect on $\mathrm{Jv}$ of removing $\mathrm{Pi}$ from the bathing medium. In proximal convoluted tubules that were equilibrated with $\mathrm{Pi}$-containing fluids, the selective removal of $\mathrm{Pi}$ from the bathing medium had no effect on Jv (1.44 \pm 0.18 vs. $1.64 \pm 0.29$ $\mathrm{nl} / \min \cdot \min ; n=5$; paired $t$ test). Other proximal convoluted tubules were equilibrated initially with $\mathrm{Pi}$ containing fluids and then $\mathrm{Pi}$ was selectively removed first from the perfusate and secondly from both bath and perfusate. These maneuvers had no effect on Jv. Thus, phosphate in the bathing medium is not required to maintain fluid absorption rates.

In contrast to the convoluted tubule, the proximal straight tubule has lower transport rates for glucose $(17,22)$ and phosphate (23) and may be exposed to very low intraluminal concentrations of $\mathrm{Pi}$ in vivo (24, 25). We measured $\mathrm{Jv}$ and glucose absorptive fluxes in five proximal straight tubules perfused from the outset with $\mathrm{Pi}$-free solutions and bathed in normal rabbit serum. These data are summarized in Table II. The mean value of $\mathrm{Jv}$ was $0.58 \pm 0.07 \mathrm{nl} / \mathrm{mm} \cdot \mathrm{min}$ and was unchanged by the addition of $\mathrm{Pi}$ to the perfusate $(0.44 \pm 0.06 \mathrm{nl} / \mathrm{mm} \cdot \min$; paired $t$ test $)$. The glucose concentration in the perfusate was $6.7 \mathrm{mM}$ and the glucose absorptive flux averaged $7.4 \pm 0.6 \mathrm{pmol} / \mathrm{mm}$. min. These values for $\mathrm{Jv}$ and for glucose absorption are similar to those reported for this segment $(17,26)$ in the presence of normal biological fluids. The proximal straight tubule therefore differs from the proximal convoluted tubule in that these transport functions are not inhibited by the removal of Pi from the luminal perfusion fluid.

Metabolic studies. The preceding data indicate that in the presence of glycolysis, limitations on phosphate availability inhibit net sodium transport by the proximal convoluted tubule. We used suspensions of rabbit cortical tubules to determine if the removal of phosphate inhibited mitochondrial function. As shown in Fig. 3, in the presence of $12 \mathrm{mM}$ glucose, the removal of extracellular Pi from the incubation media reduced $\mathrm{QO}_{2}$ from an average value of $18.9 \pm 0.6 \mathrm{nmol} /$

TABLE II

Effect of Pi-free Perfusate on Transport Function of Proximal Straight Tubules

\begin{tabular}{cccccc}
\hline Perfusate & Bath & $\mathrm{Vi}$ & $\mathrm{Jv}$ & [Gluc]o & $\begin{array}{c}\text { Gluc absorptive } \\
\text { flux }\end{array}$ \\
\hline & & $n l / \mathrm{min}$ & $n l / \mathrm{mm} \cdot \min$ & $p \operatorname{pol} / \mathrm{nl}$ & $\mathrm{pmol} / \mathrm{mm} \cdot \mathrm{min}$ \\
& & & & & \\
I Gluc $6.7 \mathrm{mM}$ & RS & $12.99 \pm 0.31$ & $0.58 \pm 0.07$ & $6.29 \pm 0.15$ & $7.41 \pm 0.65$ \\
II Gluc $6.7 \mathrm{mM}$ & RS & $13.46 \pm 0.87$ & $0.44 \pm 0.06$ & $5.98 \pm 0.18$ & $8.37 \pm 1.57$ \\
Pi $2 \mathrm{mM}$ & & & & & \\
\hline
\end{tabular}

Values are means $\pm \mathrm{SE}$ for five proximal straight tubules whose length averaged $1.55 \pm 0.05 \mathrm{~mm}$. All tubules were studied initially with a Pi-free and secondly with a Pi-containing perfusate. Abbreviations are the same as in Table $\mathbf{I}$. 


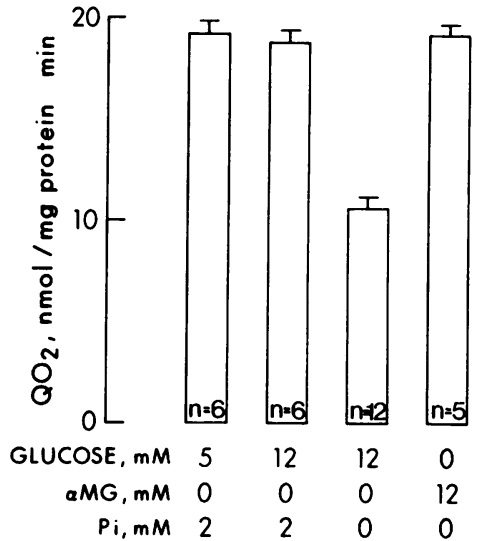

Figure 3 Effect on $\mathrm{QO}_{2}$ of hexose and $\mathrm{Pi}$ concentration. The oxygen consumption rate $\left(\mathrm{QO}_{2}\right)$ of suspensions of cortical tubules was measured in media where the concentrations of hexose or $\mathrm{Pi}$ were varied. Each bar represents the mean $\pm \mathrm{SE}$ for measurements made in $\mathrm{N}$ number of suspensions. $\alpha$ MG is the glucose analogue $\alpha$-methyl D-glucopyranoside.

$\mathrm{mg}$ protein $\cdot \min$ to $10.6 \pm 0.6 \mathrm{nmol} / \mathrm{mg}$ protein $\cdot \min$ $(P<0.001$; unpaired $t$ test). Substitution of $\alpha$-methyl $D$-glucopyranoside for $D$-glucose in the Pi-free media prevented the inhibition of $\mathrm{QO}_{2}(19.1 \pm 0.5 \mathrm{nmol} / \mathrm{mg}$ protein $\cdot \min$, Fig. 3). The inhibition of $\mathrm{QO}_{2}$ induced by the removal of extracellular $\mathrm{Pi}$ was therefore dependent on glucose metabolism. These data correspond to the reductions in fluid absorption rates shown in Table I for perfused tubules.

Oxygen consumption rates and fluid absorption rates also correspond in other experimental situations. For example, an increase in the glucose concentration from 5 to $12 \mathrm{mM}$ in the presence of extracellular Pi had no effect on $\mathrm{QO}_{2}$ (Fig. 3). In perfused tubules, Jv was unaffected by a similar increase in the intraluminal glucose concentration (Fig. 2 at $\mathrm{t}=0$ ). Additionally, in suspensions of cortical tubules, the addition of $\mathrm{Pi}$ $(2 \mathrm{mM})$ to a Pi-free medium caused only a small increase in the inhibited $\mathrm{QO}_{2}$ from $12.5 \pm 0.2$ to $13.1 \pm 0.3$ $\mathrm{nmol} / \mathrm{mg}$ protein $\cdot \min (P<0.05 ; n=5)$. As shown above in Fig. 1B, the introduction of a Pi-containing perfusate into proximal convoluted tubules that had been preequilibrated with a $\mathrm{Pi}$-free perfusate also had a minimal effect on Jv. Taken together, the data from perfused proximal convoluted tubules and from suspensions of cortical tubules suggest that in the absence of extracellular Pi, glucose metabolism induces an inhibition of oxidative metabolism and of transport in proximal convoluted tubules.

Additional studies were performed to examine the mechanism of reduced oxidative metabolism. As shown in Table III, oxygen consumption rates of cortical tu- bules were measured in three different media: $12 \mathrm{mM}$ glucose with $2 \mathrm{mM} \mathrm{Pi}, 12 \mathrm{mM}$ glucose without $\mathrm{Pi}$, and $12 \mathrm{mM} \alpha$-methyl D-glucopyranoside without Pi. Based on previous observations (27), all media contained 1 $\mathrm{mM}$ sodium butyrate to assure sufficient metabolic substrate for nystatin-stimulated mitochondrial respiration. Under these basal conditions, the average $\mathrm{QO}_{2}$ of suspensions with glucose but without $\mathrm{Pi}$ was only $45 \%$ of rates that occurred in the other two conditions. After these measurements of base-line $\mathrm{QO}_{2}$, nystatin was added to each suspension. Nystatin increases the permeability of tubular cells to sodium and potassium and thereby increases the sodium content and decreases the potassium content of the tubules. Increases in the cellular content of sodium should stimulate the activity of the $\mathrm{Na}-\mathrm{K}$ ATPase and thereby produce ADP, which in turn stimulates mitochondrial respiration (27). As shown in Table III, nystatin increased $\mathrm{QO}_{2}$ in all suspensions, although the maximal $\mathrm{QO}_{2}$ was significantly lower in the absence of phosphate. Next, an uncoupler of oxidative phosphorylation, compound 1799, was added to each suspension. This compound stimulates $\mathrm{QO}_{2}$ by dissociating mitochondrial respiration from the phosphorylation of ADP to ATP. As shown in Table III, the addition of 1799 increased $\mathrm{QO}_{2}$ in all groups of tubules but the suspensions that contained glucose without extracellular $\mathrm{Pi}$ had an average $\mathrm{QO}_{2}$ that was only $40 \%$ of rates observed in the other two conditions. Thus, in the absence of extracellular $\mathrm{Pi}$, the uncoupled rate of $\mathrm{QO}_{2}$ is impaired indicating inhibition of mitochondrial respiration. This inhibition of mitochondrial respiration would limit the capacity of tubular cells to provide ATP for the support of transport function.

TABLE III

Mitochondrial Respiration in Pi-free Media

\begin{tabular}{cccc}
\hline & \multicolumn{2}{c}{$\mathrm{QO}_{2}$ in $\mathrm{nmol} / \mathrm{mg}$ protein $\cdot \mathrm{min}$} & \\
\cline { 2 - 3 } $\begin{array}{c}\text { Media } \\
\text { composition }\end{array}$ & $\begin{array}{c}\text { Basal } \\
\text { condition }\end{array}$ & $\begin{array}{c}\text { Nystatin } \\
0.164 \mathrm{mg} / \mathrm{ml}\end{array}$ & $179910 \mu \mathrm{M}$ \\
\hline I Gluc $12 \mathrm{mM}$ & $\begin{array}{c}23.2 \pm 0.9 \\
\text { Pi } 2 \mathrm{mM}\end{array}$ & $\begin{array}{c}41.4 \pm 1.7 \\
(6)\end{array}$ & $\begin{array}{c}54.8 \pm 2.8 \\
(4)\end{array}$ \\
II Gluc $12 \mathrm{mM}$ & $\begin{array}{c}10.4 \pm 0.7^{\circ} \\
\text { Pi 0 }\end{array}$ & $\begin{array}{c}15.8 \pm 1.0^{\circ} \\
(6)\end{array}$ & $\begin{array}{c}22.1 \pm 1.9^{\circ} \\
(6)\end{array}$ \\
$\begin{array}{cccc}\text { III } \alpha \mathrm{MG} 12 \mathrm{mM} \\
\text { Pi 0 }\end{array}$ & $\begin{array}{c}23.7 \pm 0.6 \\
(5)\end{array}$ & $\begin{array}{c}41.7 \pm 1.1 \\
(5)\end{array}$ & $\begin{array}{c}53.9 \pm 0.6 \\
(3)\end{array}$ \\
\hline
\end{tabular}

All values are means $\pm \mathrm{SE}$ for a number of suspensions of cortical tubules (in parenthesis). Additions were made in the order shown along the top and were cumulative.

- Indicates values significantly different from comparable values in group I by unpaired $t$ test. $(P<0.05)$. 


\section{DISCUSSION}

Given the importance of intracellular $\mathrm{Pi}$ to energy metabolism, the purpose of our study was to determine if inorganic phosphate entering via the brush-border transport mechanisms participates in cellular metabolism. The data indicate that fluid absorption by perfused proximal convoluted tubules was eliminated by removal of $\mathrm{Pi}$ from the perfusate (Fig. 1B) and that oxygen consumption rates by suspensions of cortical tubules were markedly reduced by removal of $\mathrm{Pi}$ from the medium (Fig. 3). These effects were dependent on the metabolism of D-glucose because the effects of $\mathrm{Pi}$-free fluids could be prevented by the inhibition of glucose entry with phlorizin, by replacement of glucose with a nonmetabolized sugar, $\alpha$-methyl D-glucopyranoside, or by inhibition of glycolysis with 2deoxyglucose (Fig. 3, Table 1). Glucose-induced inhibition of fluid absorption and mitochondrial respiration provides evidence of a Crabtree effect in these tubules (10). Furthermore, because the Crabtree effect is regarded as an expression of limited availability of intracellular phosphate $(4,6)$, we conclude that the luminal entry of phosphate is required to provide sufficient phosphate for cellular metabolism. The data also provide evidence of coupling between glucose transport and glucose metabolism and between glucose metabolism and oxidative metabolism.

Henry Crabtree first described the phenomenon of glucose-induced inhibition of respiration in tissue slices from mouse sarcomas and carcinomas (28). This phenomenon also occurs in several normal tissues (10) and appears to result from competition between glycolysis and mitochondrial respiration for common intermediates or cofactors $(4,29)$. ADP, Pi, and ATP/ $\mathrm{ADP} \times \mathrm{Pi}$ have been proposed as being these intermediates $(4-6,10)$. Although its precise mechanism remains uncertain, studies of the Crabtree effect and similar phenomena provide evidence that some function of intracellular Pi may regulate the rates of glycolysis (30-32) and of mitochondrial respiration $(31,32)$.

The intracellular content of $\mathrm{Pi}$ reflects the net effect of $\mathrm{Pi}$ transport into and out of the cells as well as rates of $\mathrm{Pi}$ incorporation and release from organic compounds. Removal of $\mathrm{Pi}$ from the perfusate reduces $\mathrm{Pi}$ entry and could conceivably enhance the leakage of intracellular $\mathrm{Pi}$ into the lumen. The loss of cellular $\mathrm{Pi}$ into the perfusate, however, is not the only factor that leads to impaired cellular function in the present studies because tubular function was preserved despite $\mathrm{Pi}$ free perfusate if glucose metabolism was inhibited (Fig. 3, Table I). This suggests that reductions in available intracellular $\mathrm{Pi}$ may occur through changes in rates in organification and that these changes may be at least as important as possible losses via efflux. The possible contribution of $\mathrm{Pi}$ entering from the basolateral surface seems inconsequential because $2 \mathrm{mM}$ phosphate in the bathing medium was inadequate to preserve tubular function in the conditions of this study (Fig. 1B, and 2). The maintenance of adequate intracellular $\mathrm{Pi}$ therefore appears to be a function of $\mathrm{Pi}$ entry at the brush-border membrane, which is also responsible for the initiation of transepithelial absorption of phosphate.

The response of proximal convoluted tubules to the removal of intraluminal $\mathrm{Pi}$ depended on the protocol (Fig. 1). Net fluid absorption was absent in only those tubules exposed to $\mathrm{Pi}$-free intraluminal fluid from the outset of perfusion. On the other hand, tubules equilibrated in the presence of intraluminal phosphate absorbed fluid despite the subsequent removal of phosphate. Although speculative, we interpret these observations to indicate that intracellular phosphates, organic and inorganic, are depleted during the periods of warm and cold ischemia associated with the isolation of single tubules. This depletion of phosphates is either more extensive for tubules equilibrated with $\mathrm{Pi}$ free fluid or is not restored because of the continued absence of intraluminal phosphate. Measurements of intracellular phosphates might clarify this point but current methods lack sufficient sensitivity to detect changes in such small amounts of tissue. For proximal convoluted tubules studied by the protocol in Fig. 1B, the losses of intracellular phosphates appear to be so extensive as to prevent full recovery of cellular metabolism and transport even after perfusion for $60 \mathrm{~min}$ with fluids that contain phosphate. This observation indicates that the mitochondrial defects described below are not readily reversible or that $\mathrm{Pi}$ entry into energy-depleted cells across either cellular membrane is very limited.

The intracellular availability of $\mathrm{Pi}$ may also be affected by glucose metabolism via net incorporation of $\mathrm{Pi}$ into organic compounds. In Ehrlich ascites tumor cells, the phosphorylation of glucose by hexokinase consumes ATP and produces glucose-6-phosphate and ADP. ADP then stimulates mitochondrial respiration, which uses intracellular Pi to form ATP. Thus, as glycolysis occurs, the cytosolic content of $\mathrm{Pi}$ is reduced $(5,30)$. If cytosolic $\mathrm{Pi}$ in ascites tumor cells is reduced below $0.5 \mu \mathrm{moles} / \mathrm{g}$ wet cells, the rates of glycolysis and mitochondrial respiration are reduced and remain low until $\mathrm{Pi}$ is released from intermediates of glycolysis $(5,6)$. In the human erythrocyte, a reduction in the intracellular concentration of $\mathrm{Pi}$ caused by reduced extracellular $\mathrm{Pi}(33,34)$ inhibits glycolysis at the glyceraldehyde-3-phosphate dehydrogenase step and causes a marked accumulation of triose phosphates (35). The 
cellular content of ATP in both erythrocytes and ascites tumor cells is reduced if intracellular $\mathrm{Pi}$ falls $(5,35)$.

In our study, the oxygen consumption data indicate that oxidative metabolism is reduced by glucose metabolism if extracellular phosphate is absent (Fig. 3). This phenomenon is analogous to the Crabtree effect and thereby implies a reduction in available intracellular $\mathrm{Pi}$. Mitochondria require inorganic phosphate for several functions including oxidative phosphorylation and the uptake of substrates across mitochondrial membranes. For liver mitochondria, oxidative phosphorylation has an apparent $K_{\mathrm{m}}$ for Pi of $0.8 \mathrm{mM}$ (36). For mitochondrial membrane transport processes that involve $\mathrm{Pi}$ and other solutes such as protons, calcium, and metabolic substrates $(37,38)$, the apparent $K_{\mathrm{m}}$ are $\sim 2 \mathrm{mM}(37,38)$. If similar values apply for renal mitochondria, it is conceivable that reductions in intracellular Pi may occur and may be sufficient to impair mitochondrial respiration or oxidative phosphorylation. As shown in Table III, mitochondrial respiration was depressed to $\sim 45 \%$ of control values by conditions that presumably reduce intracellular $\mathrm{Pi}$, i.e., glucose metabolism in the absence of extracellular phosphate. Moreover, both the coupled and uncoupled rates of oxygen consumption were reduced. These observations indicate that mitochondrial respiration per se is inhibited although the mechanism cannot be localized further. Also, we cannot distinguish between a partial reduction in mitochondrial respiration in all tubules or a complete inhibition in only some tubules because removal of intraluminal $\mathrm{Pi}$ did not inhibit transport rates in proximal straight tubules (Table II).

The present data also provide indirect evidence for a relationship between glucose absorption and glucose metabolism in perfused proximal convoluted tubules. As shown in Fig. 2, in the absence of intraluminal $\mathrm{Pi}$, we could inhibit Jv by increasing glucose delivery and thereby glucose absorption to maximal rates. By analogy to the Crabtree effect, these results suggest that increased glucose transport stimulated glucose metabolism and thereby lowered intracellular Pi. Additionally, as shown in Table $I$, the reduction in fluid absorption rate associated with a Pi-free perfusate could be prevented simply by reducing glucose transport across the brush-border membrane with intraluminal phlorizin. This result suggests that glucose metabolism is reduced if glucose entry is inhibited at the brush-border membrane. From these observations, we conclude that the rate of luminal entry of glucose affects the amount of glucose involved in metabolism, presumably glycolysis. The present studies do not provide any information on what proportion of transported glucose may be metabolized nor is there infor- mation from these studies on the relative contribution of glycolysis to overall metabolism of the proximal tubule. Indeed, our observation that 2-deoxyglucose had no effect on net sodium transport measured in the presence of lactate and phosphate indicates that full rates of glycolysis are not essential to energize transport.

An interrelationship between glucose metabolism and glucose transport has been difficult to examine in vivo because oxidation of glucose is a small fraction (5-10\%) of a large oxygen consumption rate and because metabolized glucose is such a small portion (5$10 \%$ ) of the total glucose presented to the kidney (39). In vitro studies, however, suggest a positive relationship between glucose transport and metabolism. In isolated perfused rat kidney with $5 \mathrm{mM}$ glucose as the only metabolic substrate, glucose consumption rates averaged $132 \mu \mathrm{mol} / \mathrm{h}$ per $\mathrm{g}$ dry weight of tissue, and lactate formation accounted for $17 \%$ of the glucose consumed (40). When the glucose concentration in the perfusion medium was increased from 5 to $10 \mathrm{mM}$, lactate formation increased and the estimated rate of glycolysis rose by $62 \%(40)$. In this regard, Tune and Burg (17) showed that intracellular glucose averaged $14.5 \mathrm{mM}$ in perfused rabbit proximal convoluted tubules but only $4.8 \mathrm{mM}$ in nonperfused tubules. These studies along with the present data thus provide indirect evidence that increased glucose entry across the brush-border membrane may increase both the intracellular content and metabolism of glucose.

These data indicate a relationship between glucose transport and glucose metabolism, but the intracellular events that regulate glucose metabolism are unknown. Hexokinase activity in rabbit proximal convoluted tubules in vitro is relatively low and saturates at $0.5 \mathrm{mM}$ glucose (41). If the glycolytic rate were limited by hexokinase activity, an increase in the intracellular glucose concentration above $0.5 \mathrm{mM}$ such as occurs in perfused tubules (17) should have little effect on glucose metabolism. Accordingly, our observation that increases in glucose transport may stimulate glucose metabolism suggests that the hexokinase activity may not be the rate-limiting step for glycolysis in intact tubules. In this regard, data from cortical slices of rat kidney (42) also suggest that the control of glycolysis is not limited to the hexokinase reaction but may occur at several sites in the glycolytic pathway.

In this study, 2-deoxyglucose in the bath protected tubular function from the effects of a phosphate-free intraluminal fluid (Table I). Studies in the rabbit indicate that 2-deoxyglucose enters renal tubular cells (20) and studies in the rat show that after phosphorylation, 2-deoxyglucose inhibits glycolysis at the phosphoglucose isomerase step (21). In Ehrlich ascites tu- 
mor cells $(6,10), 2$-deoxyglucose is phosphorylated by hexokinase, and in the absence of glucose, causes a Crabtree effect. On the other hand, inhibition of glycolysis by iodoacetate prevents the Crabtree effect (6). In these studies, 2-deoxyglucose was added in the presence of glucose and preserved function in $\mathrm{Pi}$-free fluids, presumably by inhibition of glycolysis. 2-Deoxyglucose had no effect on glucose transport. Thus, inhibition of glucose transport or glucose metabolism via phlorizin, $\alpha$-methyl D-glucopyranoside, or 2deoxyglucose protected tubular transport and oxidative metabolism from limited phosphate availability. These observations provide strong indication that rates of glucose metabolism affect the tubular requirement for intraluminal $\mathrm{Pi}$.

Fluid absorption rates of proximal straight tubules were not inhibited by exposure to Pi-free perfusates (Table II). Proximal straight tubules differ quantitatively from proximal convoluted tubules in both transport and metabolism $(17,23,26,41)$. Accordingly, straight tubules may not be so dependent on intraluminal phosphate availability because they have lower rates of glucose absorption and metabolism, or because they may sustain smaller losses of intracellular $\mathrm{Pi}$ in response to $\mathrm{Pi}$-free perfusates. The present data do not distinguish among these and other possibilities. Because convoluted and straight portions of the proximal tubule respond differently to removal of $\mathrm{Pi}$ from the perfusate, we assume that the measurements made in suspensions of cortical tubules represent the net effect of some tubules that are affected by Pi-removal and some tubules that are not. It would therefore be difficult to interpret quantitative changes in the intracellular content of Pi and ATP measured in tubular suspensions because of the different response of convoluted and straight tubules.

We conclude that the luminal entry of phosphate provides $\mathrm{Pi}$ for cellular metabolism and that the intracellular requirement for $\mathrm{Pi}$ is affected by glucose transport and its subsequent metabolism. Increases in the rate of glucose entry into tubular cells increases the requirement for intracellular phosphate. In the absence of intraluminal phosphate, glucose metabolism causes a reduction in mitochondrial respiration and tubular function, a response analogous to the Crabtree effect.

\section{ACKNOWLEDGMENTS}

The authors acknowledge the excellent technical assistance of LaRue D. Poe and Lauren Patton and the secretarial support of Sharon D. Lee.

Dr. Brazy is a Clinical Investigator for the Veterans Administration. Dr. Dennis is a recipient of a U.S. Public Health Service Research Career Development award AM 384.
These investigations were supported by U. S. Public Health Service grants AM26201 and AM26816 and the Veterans Administration.

\section{REFERENCES}

1. Uyeda, K., and E. Racker. 1965. Regulatory mechanisms in carbohydrate metabolism. VII. The regulatory function of phosphate in glycolysis. J. Biol. Chem. 240: 46894693.

2. Passonneau, J. V., and O. H. Lowry. 1962. Phosphofructokinase and the Pasteur effect. Biochem. Biophys. Res. Commun. 7: 10-15.

3. Wilson, D. F., C. S. Owen, and A. Holian. 1977. Control of mitochondrial respiration: a quantitative evaluation of the roles of cytochrome c and oxygen. Arch. Biochem. Biophys. 182: 749-762.

4. Sussman, I., M. Erecinska, and D. F. Wilson. 1980. Regulation of cellular metabolism in the Crabtree effect. Biochim. Biophys. Acta. 591: 209-223.

5. Maitra, P. K., and B. Chance. 1965. ADP and Pi control in ascites tumor cells. In Control of Energy Metabolism. B. Chance, R. Estabrook, and J. Williamson, editors. Academic Press Inc., New York. 157-175.

6. Koobs, D. H. 1972. Phosphate mediation of the Crabtree and Pasteur effects. Science (Wash., DC). 178: 127-133.

7. Burch, H. B., O. H. Lowry, L. Meinhardt, P. Max, Jr., and K. J. Chyu. 1970. Effect of fructose, dihydroxyacetone, glycerol and glucose on metabolites and related compounds in liver and kidney. J. Biol. Chem. 245: 2092-2102.

8. Morris, R. C., Jr., K. Nigon, and E. B. Reed. 1978. Evidence that the severity of depletion of inorganic phosphate determines the severity of the disturbance of adenine nucleotide metabolism in the liver and renal cortex of the fructose-loaded rat. J. Clin. Invest. 61: 209-220.

9. Burch, H. B., S. Choi, C. N. Dence, T. R. Alvey, B. R. Cole, and O. H. Lowry. 1980. Metabolic effects of large fructose loads in different parts of the rat nephron. $J$. Biol. Chem. 255: 8239-8244.

10. Ibsen, K. H. 1961. The Crabtree effect: a review. Cancer Res. 21: 829-841.

11. Dennis, V. W., and P. C. Brazy. 1978. Sodium, phosphate, glucose, bicarbonate and alanine interactions in the isolated proximal convoluted tubule of the rabbit kidney. J. Clin. Invest. 62: 387-397.

12. Chen, R. F. 1967. Removal of fatty acids from serum albumin by charcoal treatment. J. Biol. Chem. 242: 173181.

13. Imai, M., and J. P. Kokko. 1972. Effect of peritubular protein concentration of reabsorption of sodium and water in isolated perfused proximal tubules. J. Clin. Invest. 51: 314-325.

14. Brazy, P. C., R. S. Balaban, S. R. Gullans, L. J. Mandel, and V. W. Dennis. 1980. Inhibition of metabolism: relative effects of arsenate on sodium, phosphate, and glucose transport by rabbit proximal tubule. J. Clin. Invest. 66: 1211-1221.

15. Cheng, L., and B. Sacktor. 1981. Sodium gradient-dependent phosphate transport in renal brush border membrane vesicles. J. Biol. Chem. 256: 1556-1564.

16. Balaban, R. S., S. P. Soltoff, J. M. Storey, and L. J. Mandel. 1980. Improved renal cortical tubule suspension: spectrophotometric study of oxygen delivery. Am. J. Physiol. 238: F50-F59. 
17. Tune, B. M., and M. Burg. 1971. Glucose transport by proximal renal tubules. Am. J. Physiol. 221: 580-585.

18. Brazy, P. C., and V. W. Dennis. 1978. Characteristics of glucose-phlorizin interactions in isolated proximal tubules. Am. J. Physiol. 234: F279-F286.

19. McKeown, J. W., P. C. Brazy, and V. W. Dennis. 1979. Intrarenal heterogeneity for fluid, phosphate and glucose absorption in the rabbit. Am. J. Physiol. 237: F312F318.

20. Kleinzeller, A., J. Kolinska, and I. Benes. 1967. Transport of monosaccharides in kidney-cortex cells. Biochem. $J$. 104: $852-860$.

21. Wick, A. N., D. R. Drury, H. J. Nakada, and J. B. Wolfe. 1957. Localization of the primary metabolic block produced by 2-deoxyglucose. J. Biol. Chem. 224: 963-969.

22. Barfuss, D. W., and J. A. Schafer. 1981. Differences in active and passive glucose transport along the proximal nephron. Am. J. Physiol. 241: F322-F332.

23. Dennis, V. W., P. B. Woodhall, and R. R. Robinson. 1976. Characteristics of phosphate transport in isolated proximal tubule. Am. J. Physiol. 231: 979-985.

24. Trohler, U., J-P. Bonjour, and H. Fleisch. 1976. Inorganic phosphate homeostasis. Renal adaptation to the dietary intake in intact and thyroparathyroidectomized rats. J. Clin. Invest. 57: 264-273.

25. Steele, T. H., and H. F. DeLuca. 1976. Influence of dietary phosphorus on renal phosphate reabsorption in the parathyroidectomized rat. J. Clin. Invest. 57: 867-874.

26. Burg, M. B., and J. Orloff. 1968. Control of fluid absorption in the renal proximal tubule. J. Clin. Invest. 47: 2016-2024.

27. Harris, S. I., R. S. Balaban, L. Barrett, and L. J. Mandel 1981. Mitochondrial respiratory capacity and $\mathrm{Na}^{+}-$and $\mathrm{K}^{+}$-dependent adenosine triphosphatase-mediated transport in the intact renal cell. J. Biol. Chem. 256: 1031910328.

28. Crabtree, H. G. 1929. Observations on the carbohydrate metabolism of tumors. Biochem. J. 23: 536-545.

29. Belitzer, W. A. 1936. Uber die umgekehrte Pasteursche Reaktion. Biochem. Z. 283: 330-342.

30. Wu, R., and E. Racker. 1959. Regulatory mechanisms in carbohydrate metabolism. III. Limiting factors in glycolysis of ascites tumor cells. J. Biol. Chem. 234: 10291035.

31. Wu, R., and E. Racker. 1959. Regulatory mechanisms in carbohydrate metabolism. IV. Pasteur effect and Crabtree effect in ascites tumor cells. J. Biol. Chem. 234: 1036-1041.

32. Ibsen, K. H., E. L. Coe, and R. W. McKee. 1960. Some factors influencing respiration and glycolysis in Ehrlich ascites tumor cells. Cancer Res. 20: 1399-1407.

33. Vestergaard-Bogind, B., and T. Hesselbo. 1960. The transport of phosphate ions across the human red cell membrane. I. The distribution of phosphate ions in equilibrium at comparatively high phosphate concentrations. Biochim. Biophys. Acta. 44: 117-130.

34. Deuticke, B. 1970. Anion permeability of the red blood cell. Naturwissenschaften. 57: 172-179.

35. Travis, S. F., H. J. Sugerman, R. L. Ruberg, S. J. Dudrick M. Delivoria-Papadopoulos, L. D. Miller, and F. H. Oski. 1971. Alterations of red cell glycolytic intermediates and oxygen transport as a consequence of hypophosphatemia in patients receiving intravenous hyperalimentation. $N$. Engl. J. Med. 285: 763-768.

36. Ernster, L., C. P. Lee, and S. Tanda. 1966. The reaction sequence in oxidative phosphorylation. In Biochemistry of Mitochondria. E. C. Slater, Z. Kaniuga, and L. Wojtczak, editors. Academic Press Inc., New York. 2951.

37. LaNoue, K. F., and A. C. Schoolwerth. 1979. Metabolite transport in mitochondria. Ann. Rev. Biochem. 48: 871922.

38. Fonyo, A. 1979. Inhibitors of mitochondrial phosphate transport. Pharmacol. Ther. 7: 627-645.

39. Cohen, J. J., and M. Barac-Nieto. 1973. Renal metabolism of substrates in relation to renal function. V. Carbohydrate metabolism in the kidney. In Handbook of Physiology, Section 8: Renal Physiology. J. Orloff and R. W. Berliner, editors. American Physiological Society, Washington, DC. 27: 961-1001.

40. Ross, B. D., F. H. Epstein, and A. Leaf. 1973. Sodium reabsorption in the perfused rat kidney. Am. J. Physiol. 225: 1165-1171.

41. Vandewalle, A., G. Wirthensohn, H. G. Heidrich, and W. G. Guder. 1981. Distribution of hexokinase and phosphoenolpyruvate carboxykinase along the rabbit nephron. Am. J. Physiol. 240: F492-F500.

42. Wu, R. 1965. Rate limiting factors in glycolysis and inorganic orthophosphate transport in rat liver and kidney slices. J. Biol. Chem. 240: 2373-2381. 